\title{
Text Fragment Recovery Method Based on Imperial Competition Algorithm
}

\author{
Dongyu Guo ${ }^{\text {a) }}$, Zeyuan Tao ${ }^{\text {b) }}$ \\ A College of Automation, Wuhan University of Technology, Wuhan 430070, China \\ a) Corresponding author: guodongyu1209@163.com \\ b) tzy@whut.edu.cn
}

\begin{abstract}
In this paper, a text fragment splicing method based on K-means and Empire competition algorithm is proposed. First, use K-means clustering to find out a piece of paper that belongs to one line; Then, define the edge distance of the paper, and establish a combined optimization model; using the imperial competition algorithm to optimize the solution. The experimental results show that the speed and accuracy of this algorithm are improved compared with the literature algorithm and have certain application value.
\end{abstract}

Key words: Imperial Competition Algorithm; K-MEANS clustering; text fragment splicing.

\section{INTRODUCTION}

Text fragment splicing technology has a strong applicability in the field of historical document restoration and military intelligence restoration. Images and recovery is a typical application in the field of computer vision. In the computer field, the existing text fragment splicing method has simulated annealing algorithm [1]; Genetic algorithm [2]; Ant Colony Algorithm [3], etc. But when there are too many text fragments, the classic optimization algorithm is not enough to get the optimal solution. This paper presents a method of text fragment restoration based on imperialist competitive algorithm.

The Imperial Competition Algorithm (ICA) was proposed by Atashpaz-Gargari and Lucas in 2007. The Imperial Competition Algorithm is applied to solving TSP [1], power system design [2]et.

This paper based on the existing research, proposes a discrete ICA algorithm to solve the problem of text segment splicing. First, import the text fragment image information into the computer. Then, the image is binarized. transform the binary image into a matrix for clustering. Finally, according to the discrete ICA algorithm, the overall matching degree is the highest means the restored image is obtained. After many simulation experiments, the results are compared with other algorithms, and the feasibility and effectiveness of the algorithm in the splicing of fragments are proved.

\section{RESTORATION OF TEXT FRAGMENTS BASED ON ICA}

\section{Convert Image Information into a Matrix.}

The text fragments used in this paper have the same size and shape, and they are all $\mathrm{p} \times \mathrm{q}$ pixels. First, the text image is imported and stored in the computer. In order to facilitate the further processing, the text fragment image is transformed into grayscale image, and the binary image of black word white background is further obtained. In the actual situation, the image of text fragment image binarization is shown in figure 1. 


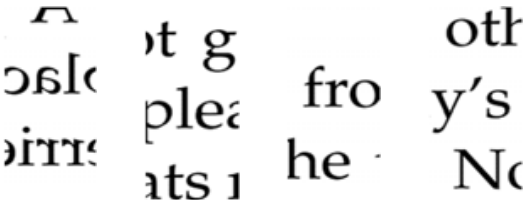

FIGURE 1. Text fragments example

The binary image information is stored in the computer as $\mathrm{p} \times \mathrm{q}$ size, $0-1$ matrix $\mathrm{S}$,

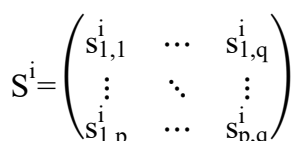

\section{Picture K- mean Clustering}

Since the original text segment image is cut in two directions on the two-dimensional plane, the same row can be clustered first using k-means clustering to simplify the subsequent calculation. The steps are as follows:

Step1: Select $m$ text segments as cluster centers $Z_{1}(1), Z_{2}(1), \cdots, Z_{m}(1)$, and the parenthetical numbers in the parentheses represent the number of iterations of the cluster center.

Step2: Use the shortest distance principle to assign the sample to one of the m cluster centers, if

$$
\min \left\{\left\|X-Z_{i}(k)\right\|, i=1,2, \cdots, m\right\}=\left\|X-Z_{j}(k)\right\|=D_{j}(k)
$$

Then $\mathrm{X} \in \mathrm{S}_{\mathrm{j}}(k)$.In the formula, $\mathrm{k}$ is the sub ordinal number of the iterative operation.

Step3: Calculate the new vector value of each cluster center. $Z_{\mathrm{j}}(k+1), j=1,2, \cdots, m$.

$$
\mathrm{Z}_{\mathrm{j}}(\mathrm{k}+1)=\frac{1}{\mathrm{~N}_{\mathrm{j}}} \sum_{\mathrm{X} \in \mathrm{S}_{\mathrm{j}}(\mathrm{k})} \mathrm{X}, \mathrm{j}=1,2, \cdots, \mathrm{m}
$$

Then use the average vector as a new clustering center. In this step, we need to calculate the sample mean vectors of $\mathrm{M}$ clusters separately.

Step4: If $\mathrm{Z}_{\mathrm{j}}(k+1) \neq \mathrm{Z}_{\mathrm{j}}(k), j=1,2, \cdots, m$, then back to step 2 , reclassify the pattern samples one by one, and iterate over the calculation. If $\mathrm{Z}_{\mathrm{j}}(k+1)=\mathrm{Z}_{\mathrm{j}}(k), j=1,2, \cdots, m$, algorithm convergence, clustering finished.

\section{The Establishment of the Text Fragments Splicing Model.}

After k-means clustering, each line of the fragment is analyzed and it is found that the proximity index can be set as the matching degree of the image edge pixels. The reference [3] provides a solution model for the splicing of scrap paper. The goal of stitching is to minimize the value of the edge gap of each sub image in the combination of images based on stitching order. The concrete mathematical expression of this model is as follows:

$$
\begin{aligned}
& \operatorname{minC}=\sum_{i=1}^{n} \sum_{j=1}^{n} \operatorname{dis}_{\mathrm{ij}} \mathrm{x}_{\mathrm{ij}} \\
& \text { st. } \sum_{\mathrm{j}=1}^{\mathrm{n}} \mathrm{x}_{\mathrm{ij}}=1, \mathrm{i}=1,2, \cdots, \mathrm{n}
\end{aligned}
$$




$$
\begin{gathered}
\sum_{\mathrm{i}=1}^{\mathrm{n}} \mathrm{x}_{\mathrm{ij}}=1, \mathrm{j}=1,2, \cdots, \mathrm{n} \\
\sum_{\mathrm{i}, \mathrm{j} \in \mathrm{s}} \mathrm{x}_{\mathrm{ij}} \leq|s|_{-1,2 \leq}|s|_{\leq \mathrm{n}-1}
\end{gathered}
$$

In $x_{i j} \in\{0,1\}, i, j=1,2, \cdots n, i \neq j, x_{i j}$ is $0-1$ variable, when it is equal to 1 , it means that the paper $i$ is splice into the front of the paper $j$, otherwise it does not splice. The last three main constraints said each fragment will only to a piece of paper, each piece will only spell ahead in front of a piece of paper, in addition to the fore and aft two pieces, other paper does not constitute a loop. Through the analysis of the above mathematical models, we can find that the mathematical model of the problem is consistent with the traveling salesman problem model and belongs to the asymmetric traveling salesman problem.

\section{ICA for Solving the Segmentation of Text Fragments}

\section{Coding}

First, encode the original Empire. The coding methods of TSP include path representation, sequential representation and neighbor representation. In this paper, the neighborhood representation is chosen to encode. Each initial empire can be regarded as a solution to this problem. Taking ten texts as an example, and the text fragments in turn are 5-8-1-9-2-10-6-3-7-4.

\section{Initialization of the Empire}

In the period of the initialization of the Empire, random generation of $\mathrm{K}$ initial states, calculated the normalized cost value $\mathrm{C}_{\mathrm{i}}$ of each country, the normalized cost value of each country is calculated by the maximum image edge gap value of all solutions minus image edge gap value of this current solution. That is $C_{i}=\max \left\{c_{n}\right\}-c_{i} ; c_{i}$ is the cost value of each country, that is the image edge gap of the current solution. According to the national standardized cost value, all countries are arranged in descending order. Selecting the top Nimp countries as colonial countries, the number of colonies $N$ col $=N$ pop $-N$ imp.

Then calculate the power of each colonial country. The number of colonies allocated by each colonial country is related to the power of the colonial power.

$$
\mathrm{P}_{\mathrm{i}}=\mathrm{c}_{\mathrm{i}} / \sum_{\mathrm{i}=1}^{\mathrm{N}_{\mathrm{imp}}} \mathrm{c}_{\mathrm{i}}
$$

The number of colonies assigned to it $\mathrm{NC}_{\mathrm{i}}=\operatorname{round}\left\{\mathrm{P}_{\mathrm{i}} \times \mathrm{N}_{\text {col }}\right\}$, and $\mathrm{Pi}$ is the power value of the colonials' power. Round function is rounded to the integral function.

\section{Colonial assimilation}

Assimilation is a process of internal colonial empire gradually tends to colonial countries, this paper takes the following four steps to generate new assimilation colonies.

Step1: The probability of randomly generating between 0 and 1 in each urban coding position in the colony.

Step2: In the colonial location where all probability is greater than or equal to $\rho$, the colonial city code of that location is used as assimilate colony number.

Step3: For the location of the colony with smaller probability that $\rho$, if the city number at the location does not appear at the rest of the colonies, the city number of the colonies in this position is used as the number of the assimilation colonies. Step2 and Step3 are collectively referred to as the replacement process.

Step4: The process of reconstruction. For the city number that is not appearing in the assimilation colony, the increase distance to all possible locations in the assimilative colonial city sequence is calculated in turn, and the city 
number is inserted at the minimum increase distance. The increase distance $\mathrm{N}$ of inserting the city numbered $i$ into the city code number $a$ and $b$ adjacent position is $\mathrm{N}=\operatorname{dis}(\mathrm{a}, \mathrm{i})+\operatorname{dis}(\mathrm{i}, \mathrm{b})-\operatorname{dis}(\mathrm{a}, \mathrm{b})$.

dis $(a, i)$ represents the distance between $a$ cities and $i$ cities. After the assimilation process is completed, the original colony is replaced by the newly generated assimilation colony.

Step5: Calculate the power value of the new colony, if it is superior to the colonial country it belongs to, will replace the solution of the colonial country. The specific process is shown in Figure 2. $\rho$ is taken 0.5.

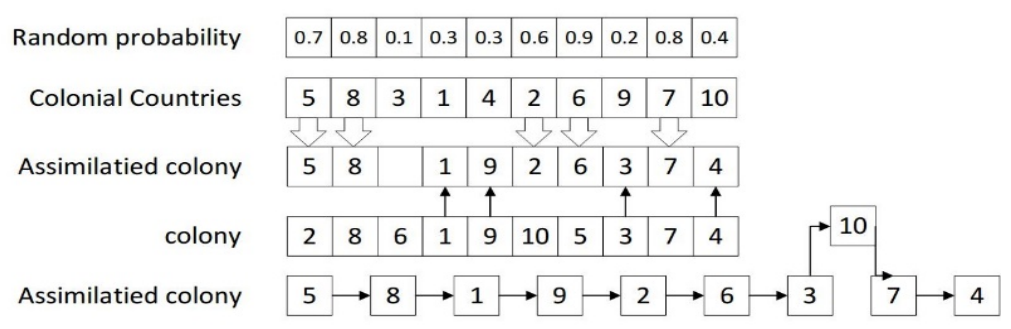

FIGURE 2. Assimilation process using ten text fragments as an example

\section{Colonial revolution}

In this paper, the colonial revolution was set up as exchange the number of two random locations. This can avoid invalidation of random search while giving full play to the mechanism of revolution to maintain the function of population diversity. Establishing a formula of adjustment from the distance between colonial and colonial countries to the probability of revolution $\mathrm{PT}_{\mathrm{i}}$.

$$
\mathrm{PT}_{\mathrm{i}}=\eta \times \operatorname{dist}(\mathrm{i}) / \sum_{\mathrm{i}=1}^{\mathrm{NC}} \operatorname{dist}(\mathrm{i})+(1-\eta) \times \operatorname{rand}(0,1)
$$

dist.(i) is the distance between colony and colonial countries. ci represents the cost of the i colony; ck represents the cost of the k colony; dist.(i) is defined as dist $(\mathrm{i})=\mathrm{C}_{\mathrm{i}}-C_{k}$. When the probability of revolution $\mathrm{PT}_{\mathrm{i}}$ is greater than the given threshold $\mu$, revolute the colony. According to the requirement of revolution, when the empire is far away from the colony, that is, when the solution of the colony is relatively poor, the probability of its revolution will increase. The specific process of the colonial revolution is as follows:

Step1: Random generation of $\alpha, \beta$,And satisfy $\alpha, \beta \in(1, m \times n)$.

Step2: Interchanging two coded numbers of $\alpha, \beta$ position in the colony, create a new colony

Step3: Calculating the power value of the new colony, if it is superior to the colonial country to which it belongs, will replace the solution of the colonial state.

\section{Imperial competition and parameter selection in the algorithm}

Imperial competition is the key step of algorithm convergence, which originated from the social behavior of colonial redistribution. Specific operation steps are shown in the reference [1], [4].

ICA has three important parameters: the total number of countries, the number of colonial countries, and the threshold of colonial revolution probability. A large number of case studies show that the algorithm performs best when the number of colonial countries accounts for $10 \% \sim 20 \%$ of the state. Based on a large number of experiments, the best parameters of ICA are $\mathrm{N}=100, \mathrm{~N}_{\mathrm{im}}=15, \mu=0.3$. When using these parameters, ICA has the best performance.

\section{Algorithm Verification and Result Analysis}

The characteristics of noise sensitivity, time consuming and effectiveness are very important in the problem of text stitching. In order to verify the characteristics of the algorithm, MATLAB programming is used to implement the above algorithm, and verified and compared by reference [3]. 
The examples of the application of the test picture in reference [3] are $10 * 15$ and $10 * 12$. The test is runs on the 4.0GB RAM 2.20GHz computer, programmed by Matlab2015b.

In order to analyze and compare the running effect of ICA algorithm on the problem of text fragment splicing, it runs independently 20 times. Calculate each run time and take 20 running time averages.

TABLE 1. The time-consuming comparison results

\begin{tabular}{cccc}
\hline & Lingo [3] & ACO [3] & ICA \\
\hline The Maximum time (S) & 98.503 & 5.981 & 5.029 \\
The minimum time (S) & 93.617 & 5.268 & 4.422 \\
The average time (S) & 95.545 & 5.691 & 4.781 \\
\hline
\end{tabular}

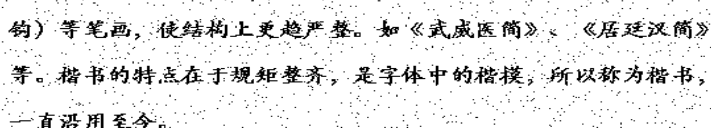

FIGURE 3. The row images after K-means clustering
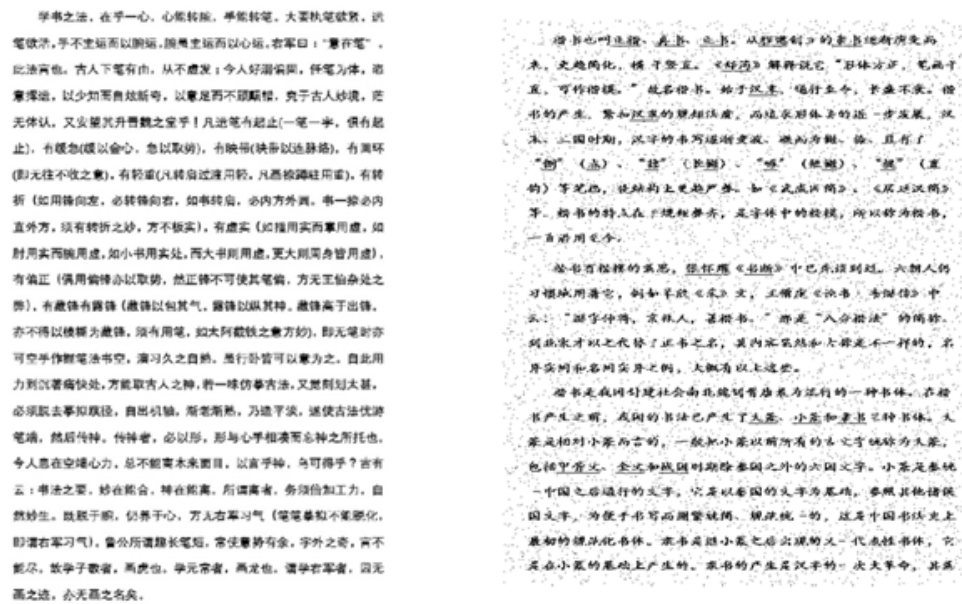

FIGURE 4. Assimilation process using ten text fragments as an example

Fig. 3 is an image that is clustered into a row using the k-means method, but the method needs manual correction. Figure 4, Figure 5, successfully restored the image, verifying the effectiveness of the algorithm. The image with salt and pepper noise can also be restored successfully, which shows that the algorithm is robust. We can see that the algorithm is more efficient in comparison of timeliness.

\section{CONCLUSION}

In this paper, a new method based on improved discrete ICA algorithm is proposed to solve the problem of text fragments mosaicking. use two value method to extract of fragment image information. Clustering fragments into rows using k-means clustering. Using the matching degree of the edge information of each line fragment as a measure of whether or not the debris is adjacent or not, a restoration model with the best overall matching degree is established. The improved discrete ICA algorithm is used for encoding and solving. Experimental results show that the discrete ICA algorithm used in this paper is a practical and feasible algorithm to solve the text fragment restoration model. The algorithm has good stability, high efficiency and strong robustness. 


\section{REFERENCES}

1. Nemati K, Shamsuddin S M, Kamarposhti M S. Using Imperial Competitive Algorithm for Solving Traveling Salesman Problem and Comparing the Efficiency of the Proposed Algorithm with Methods in Use[J]. Australian Journal of Basic \& Applied Sciences, 2011, 5(10).

2. Gharavi H, Ardehali M M, Ghanbari-Tichi S. Imperial competitive algorithm optimization of fuzzy multiobjective design of a hybrid green power system with considerations for economics, reliability, and environmental emissions[J]. Renewable Energy, 2015, 78:427-437.

3. Zixiao Pan, Mei Wang, "A New Method of Shredded Paper Image Restoration Based on Ant Colony Algorithm," Chinese Automation Congress (CAC). Jinan: Academic, 2017, pp. 5526-5530.

4. Atashpaz-Gargari E, Lucas C. Imperialist competitive algorithm: An algorithm for optimization inspired by imperialistic competition[C]// Evolutionary Computation, 2007. CEC 2007. IEEE Congress on. IEEE, 2008:4661-4667.

5. Pan Z, Wang M. A New Method of Shredded Paper Image Stitching and Restoration[C]// International Conference on Industrial Informatics - Computing Technology, Intelligent Technology, Industrial Information Integration. IEEE Computer Society, 2017:55-58. 